\title{
Reading and the Corpus Callosum: An MRI Family Study of Volume and Area
}

\author{
Jodene Goldenring Fine and \\ Margaret Semrud-Clikeman \\ Michigan State University \\ Laura M. Stapleton \\ University of Maryland, Baltimore County
}

\author{
Timothy Z. Keith \\ University of Texas at Austin
}

George W. Hynd

Purdue University

\begin{abstract}
This study examines the corpus callosum in 68 readers nested in 24 families. Callosa were measured and controlled for whole brain volume, intelligence, and gender. The relation of corpus callosum size to the within-family variance of oral reading was investigated with various measurements: volume, midsagittal area, and anterior-to-posterior one-fifth area segmentations. Because this is the first known publication of MRI calculations of corpus callosum volume, some basic questions about bilateral symmetry and the efficacy of area versus volumetric measurements were explored. Results suggest that better readers within families have larger midsagittal areas at the midbody. Although reliably measured, volume did not contribute to oral reading but was highly correlated with area. Bilateral volumes of the corpus callosum were symmetric.
\end{abstract}

Keywords: corpus callosum, dyslexia, magnetic resonance imaging, reading disorder, family differences

Reading disorder (RD) is the most prevalent of all of learning disabilities. Estimates of incidence of children with RD vary from $5-10 \%$ (Shaywitz, 1998) to as high as $17.5 \%$ (Breier et al., 2003) of the general population. It is the diagnosis for $80 \%$ of children identified as learning disabled. Severe RD, also called dyslexia, is now known to be familial and heritable, and the result of neurobiological differences in the brain.

Current theories on the development of RD emphasize problems in phonological processing, rapid naming, magnocellular deficits in the primary visual cortex (Jenner, Rosen, \& Galaburda, 1999), interhemispheric transfer of information (Snowling, 2000), interhemispheric coordination and timing (Kinsbourne, 2003), and the development of unusual right-hemisphere reading areas not found in individuals with normal reading development (Shaywitz et al., 1998). Differences in bilateral structures such as the planum temporale have also been found (Duara et al., 1991; Hynd, SemrudClikeman, Lorys, Novey, \& Eliopulos, 1990; Rumsey et al., 1986). In addition, six studies on the size of the corpus callosum and reading have been published.

Jodene Goldenring Fine and Margaret Semrud-Clikeman, Departments of Psychology and Psychiatry, Michigan State University; Timothy Z. Keith, Department of Educational Psychology, University of Texas at Austin, Laura M. Stapleton, Department of Psychology, University of Maryland, Baltimore County, George W. Hynd, College of Education, Purdue University.

Preparation of the manuscript was supported in part by Grant NIH/ NICHD-1-R01-26890-07, awarded to George W. Hynd by the National Institutes of Health/National Institute of Child Health and Human Development, and in part by the Donald and Sybil Harrington Foundation.

Correspondence concerning this article should be addressed to Jodene Goldenring Fine, Departments of Psychology and Psychiatry, 321 West Fee Hall, Michigan State University, East Lansing, MI 48824. E-mail: finej@msu.edu
Of the studies conducted on the size of the corpus callosum in relation to dyslexia, three found areas of the corpus callosum to be greater than that of controls, although the studies do not agree on which portions of the callosum were larger. Duara et al. (1991) found a larger splenium in participants with dyslexia and in females, although gender was not controlled for in the area-bydiagnosis estimate. Participants with attention deficit hyperactivity disorder (ADHD) were included in Duara et al.'s study, but ADHD was not controlled for though differences in the corpus callosum of this group have been found (Hynd et al., 1991). Rumsey et al. (1996) removed participants with ADHD from their study and found the splenium and the isthmus together to be larger at $p<$ .02 , with whole corpus callosum area covaried and participants well matched for socioeconomic status, handedness, and IQ. Robichon and Habib (1998) also found larger callosum area in dyslexics, in the isthmus and total midsagittal area but not in the splenium. In this study of male participants only, there was no correction for whole brain size and no covariates for handedness and IQ.

Hynd et al. (1995) found that children with dyslexia had smaller areas in the genu and no differences in the splenium or other areas compared to controls. In that study of 32 children, there was more than a one standard deviation difference in the group means for IQ; however, whole-brain volume was not controlled, and children with ADHD as well as those with developmental language disorder (DLD) were included. Two studies found no differences in the size of the corpus callosum of dyslexic and normally developed readers (Larsen, Hoeien, \& Oedegaard, 1992; Njiokiktjien, de Sonneville, \& Vaal, 1994), but neither study covaried handedness or gender.

Past studies offer few substantive results regarding differences in corpus callosum morphology between dyslexic and normally developed readers. Small sample size and poor covariate control contribute to the disagreement among studies. Moreover, widely 
varying criteria for the identification of the RD groups were used, ranging from familial history of RD (e.g., Njiokiktjien, de Sonneville, \& Vaal, 1994) to specific deficiencies in reading and spelling (e.g., Rumsey et al., 1996). In his review of studies on corpus callosum morphology in dyslexics, Beaton (1997) lamented, "there is a need for a large-scale, well-controlled study in which dyslexic subjects can be considered to comprise a reasonably homogenous group with regard to the nature of the underlying deficit" (Beaton, 1997, p. 303).

\section{Study Questions}

Although RD tends to run in families, the nature of the disorder is rarely studied under familial conditions. The current study examines the variance of the corpus callosum within families. In other words, how do the corpus callosa of reading disabled members of a family compare with those of better readers in the family? The corpus callosum is known to vary widely across individuals (Beaton, 2004) and is also known to be variable in response to environmental conditions (Castro-Caldas et al., 1999; Kopcik, Juraska, \& Washburne, 1986; Schlaug, Jäncke, Huang, Staiger, \& Steinmetz, 1995; Strauss \& Wada, 1993). Its size is also known to be highly heritable (Scamvougeras, Kigar, Jones, Weinberger, \& Witelson, 2003). Thus, in families we might expect to reduce variability between participants, better revealing differences in the morphology of the corpus callosum related to reading ability.

Previous studies have analyzed the corpus callosum midsagittal area. This study also analyzed the volume of the corpus callosum, raising some basic questions. Is volume a better measurement? Are the bilateral volumes symmetrical? And finally, is area at the midsagittal slice a good estimate of volume? Similar to other studies, this study also examines anterior-to-posterior segments of the corpus callosum area at the midsagittal slice. Like other researchers, we are interested in where differences in corpus callosum size can be found in relation to reading ability, but with gender, IQ, and whole-brain volume controlled.

\section{Method}

\section{Participants}

All families with a known history of ADHD were excluded from the study, as were participants with Full Scale IQs of less than 85 on the Wechsler Abbreviated Scale of Intelligence (WASI; Psychological Corporation, 1999) and those with other neurological disorders. Sixty-eight participants nested in 24 families were included in the final sample. Of these, there were 24 children (15 male and 9 female) and 44 adults (20 male and 24 female). Six participants ( 2 children and 4 adults) were African American. The children's ages ranged from 6 to nearly 17 years old, with the mean age at 10.4 years $(S D=2.04)$. The mean age of the adults was 40.1 years and ranged from 31 to 49 years $(S D=4.22)$.

The presence of an RD was determined at the time of data collection according to the criteria used by the state of Georgia for a learning disability in reading. Participants given an RD diagnosis had a minimum 20-point standard score discrepancy between intelligence and achievement score, with intelligence greater than reading achievement, as determined by the WASI full-scale IQ score and academic achievement in reading. Reading was assessed with the Passage score obtained from the Gray Oral Reading
Test-Third Edition (GORT-3: Wiederholt \& Bryant, 1992). Some of the participants with RD, particularly the adults in the study, were at least partially compensated readers. It has been suggested that compensated readers with RD use memory to support their acquisition of large sight-word lexicons (Snowling, 2000); thus, single-word reading accuracy would not be the best measure of their degree of disability. See Table 1 for means and standard deviations of WASI and GORT-3 scores by group.

\section{Procedures}

The GORT-3 assesses both rate and accuracy in paragraph reading, and these two variables were used as dependent measures of reading skill in this study. The single score for Passage is a combined variable for GORT-3 rate and accuracy, also used in the study. Gender was included as a control variable, but lateral dominance was not used because of insufficient data collection. Table 2 shows the standard deviations and $t$ values for tests of significant differences between RD and able readers. No significant differences in WASI IQ scores were found between RD and able readers. Significant differences in GORT-3 reading scores were found between children who are able and RD readers. Adult compensated RD readers had GORT-3 scores that were not significantly different from the scores of able readers.

Acquisition and preliminary assembling of the MRI data to assess corpus callosum size were conducted at the University of Georgia at the direction of George Hynd. Three-dimensional MRI images of the brain were obtained with a 1.5 Tesla GE Signa scanner (TE [echo time] $=$ min full; flip angle $=30$; field of view $=24$; frequency and phase $=256$; frequency direction $=\mathrm{S} / \mathrm{I}$ [superior/inferior]). Gapless slices $1.5 \mathrm{~mm}$ thick were collected in the sagittal plane.

\section{Variables and Analyses}

Obtaining measurement variables. The software program Analysis of Functional NeuroImages (AFNI; Cox, 1996) was used to align the MRI images, to obtain uniform density, to identify the limits of the corpus callosum, and to calculate whole-brain volume. A five-step protocol was used to identify voxels within the corpus callosum, which was defined as the higher density band of

Table 1

Mean Scores (and Standard Deviations) for IQ and Oral Reading Among Groups

\begin{tabular}{lcc}
\hline & WASI & Full-scale IQ \\
\multicolumn{1}{c}{ Group $(n)$} & $M(S D)$ & $\begin{array}{c}\text { GORT-3 } \\
\text { Passage } \\
M(S D)\end{array}$ \\
\hline Children & & \\
$\quad$ Able (12) & $100.08(9.8)$ & $94.17(13.0)$ \\
RD (12) & $109.67(16.6)$ & $75.00(10.0)$ \\
Adults & $110.97(10.8)$ & $114.69(13.1)$ \\
Able (32) & $108.33(13.2)$ & $110.63(11.6)$ \\
RD + comp (9) & $101.67(6.0)$ & $68.33(5.8)$ \\
RD (3) &
\end{tabular}

Note. $\quad$ WASI $=$ Wechsler Abbreviated Scale of Intelligence; GORT-3 = Gray Oral Reading Test-Third Edition; RD = reading disorder; comp = compensated. 
Table 2

Comparison of Scores Among Groups

\begin{tabular}{|c|c|c|c|c|}
\hline \multirow[b]{2}{*}{ Group } & \multicolumn{2}{|c|}{$\begin{array}{l}\text { WASI full-scale } \\
\text { IQ }\end{array}$} & \multicolumn{2}{|c|}{$\begin{array}{l}\text { GORT-3 } \\
\text { passage }\end{array}$} \\
\hline & $t$ & $p$ & $t$ & $p$ \\
\hline \multicolumn{5}{|l|}{ Children } \\
\hline $\begin{array}{l}\text { Able vs. RD } \\
\text { Adults }\end{array}$ & -1.72 & .099 & 4.06 & .001 \\
\hline Able vs. RD + comp & 1.15 & .258 & 2.58 & .021 \\
\hline Able vs. comp & 0.62 & .540 & 1.37 & .180 \\
\hline RD vs. comp & -1.19 & .268 & -7.78 & .001 \\
\hline
\end{tabular}

Note. $\quad$ WASI $=$ Wechsler Abbreviated Scale of Intelligence; GORT-3 = Gray Oral Reading Test-Third Edition; RD = reading disorder; comp = compensated.

fibers directly above the lateral ventricle and thalamus and below the cingulate gyrus in all sagittal planes. Measurement reliability estimates were obtained based on the re-measurement of 15 randomly selected scans. A correlation of .935 was found for corpus callosum volume and .947 for corpus callosum area.

Controlling for brain size. To control for age and whole-brain size, the residuals of a regression of raw corpus callosum volume on age and whole-brain volume were obtained. Regressions conducted separately on child and adult groups indicated that age drops out as a significant contributor to corpus callosum volume when the effect of whole-brain volume is controlled $(\beta=.268$, $b=21.163, p=.058)$. These results suggest that the developmental effect of corpus callosum growth is largely subsumed by overall brain growth. For subsequent analyses, regression residuals were used as the measure of corpus callosum size with the effect of whole-brain volume removed, essentially controlling for brain size. These measurements are referred to as "adjusted" (i.e., "adjusted corpus callosum area"). All corpus callosum measurements (volume and area) referred to in this study were adjusted in this manner.

Anterior-posterior segmentation. Segmentation of the corpus callosum midsagittal slice into five equal areas was accomplished digitally with XYZ coordinates from AFNI brought into SPSS. When the anterior-posterior lengths were not evenly divisible by five, 100 segmentation iterations with random placement of remainders were performed, and the average calculated for each segment was used in subsequent analyses. Segments were adjusted for whole brain size.
Nonindependent sample. Intraclass correlations indicated that a portion of variance in IQ, reading, and whole-brain volume were attributable to family membership. A small intraclass correlation was found for corpus callosum volume, but no correlation was obtained once the corpus callosum was controlled for whole brain volume. Because of family membership effects, it was deemed appropriate to center the data on family means, yielding analyses of within-family variance rather than between-subjects variance. Table 3 shows the estimates of the intraclass correlations, with brain measurements in voxels. Note that even small intraclass correlations on the order of .10 can increase Type I error (Stevens, 2002).

Analyses. All corpus callosum variables were adjusted for whole-brain volume and centered on the family mean. IQ and GORT-3 scores were also centered. To examine the effects of corpus callosum size on oral reading, GORT-3 scores were regressed on IQ, gender, and corpus callosum measurements. Change in $R^{2}$ when corpus callosum measurements were added to the regression model was used as an estimate of effect size. An analysis testing the bilateral symmetry of corpus callosum volumes in individuals was performed on uncentered, raw volumes with a one-way repeated measure analysis of covariance (ANCOVA). Left and right volumes were the repeated measures, with uncentered IQ and age, gender, and diagnosis included as covariates. Finally, a Pearson correlation was used to estimate the correlation between raw area and volume measurements. The reliability estimates obtained for the study corpus callosum measurements were used to adjust for attenuation of correlation.

\section{Results}

\section{Corpus Callosum Volume}

The relation of corpus callosum volume to oral reading was analyzed first. Regression results indicate that, taken together and for this sample, IQ, gender, and total corpus callosum volume adjusted for brain volume accounted for 23 percent of the withinfamily variance in reading achievement, $R^{2}=.23, F(3,40)=6.378$, $p<.001$. Table 4 shows the regression weights for the variables. Intelligence scores appear to have the strongest influence on reading achievement $(\beta=.319)$. The unstandardized coefficients show that a one standard scale point increase in IQ score was associated with a .617 point increase in GORT-3 Passage scores. There was a small gender effect, with female participants scoring 7 points higher on reading, on average, than male participants (female

Table 3

Intraclass Correlations for Continuous Variables in the Study

\begin{tabular}{lrrr}
\hline Variable $(n .=2.83)$ & $\begin{array}{c}\text { Mean square, } \\
\text { between }\end{array}$ & $\begin{array}{c}\text { Mean square, } \\
\text { within }\end{array}$ & $\begin{array}{c}\text { Intraclass } \\
\text { correlation }\end{array}$ \\
\hline WASI full-scale IQ & 275.62 & 90.47 & .42 \\
GORT-3 passage & 557.21 & 338.45 & .19 \\
Whole-brain volume & $23,752,958,218.90$ & $1,739,138,5831.40$ & .11 \\
Raw CC volume & $4,032,588.62$ & $3,763,207.98$ & .03 \\
Adjusted CC volume & $2,019,418.77$ & $2,648,652.64$ & -.09 \\
Adjusted CC area & $7,667.69$ & $11,073.82$ & -.12 \\
\hline
\end{tabular}

Note. WASI $=$ Wechsler Abbreviated Scale of Intelligence; GORT-3 = Gray Oral Reading Test—Third Edition; $\mathrm{CC}=$ Corpus Callosum. 
Table 4

Results of the Regression of GORT-3 Passage Scores on Adjusted Corpus Callosum Volume, With IQ and Gender Controlled

\begin{tabular}{lcccc}
\hline Variable & $\begin{array}{c}\text { Unstandardized } \\
\text { coefficient } \\
(b)\end{array}$ & $\begin{array}{c}\text { Standardized } \\
\text { coefficient } \\
(\beta)\end{array}$ & $\begin{array}{c}\text { Significance } \\
(p)\end{array}$ & $\begin{array}{c}\text { Effect size } \\
\left(\Delta R^{2}\right)\end{array}$ \\
\hline Full-scale IQ & 0.617 & .319 & .008 & \\
Gender & -7.062 & -.239 & .040 & \\
CC volume & 0.001 & .130 & .257 & .016 \\
\hline
\end{tabular}

Note. GORT-3 = Gray Oral Reading Test-Third Edition; $\mathrm{CC}=$ Corpus Callosum.

participants were coded 0 and male participants were coded 1$)$. In contrast, corpus callosum volume did not contribute significantly in explaining within-family variability of oral reading scores; the change in $R^{2}$ was nonsignificant when corpus callosum volume was added to the regression model, $\Delta R^{2}=.016, F(2,39)=1.325$, $p=.254$.

\section{Corpus Callosum Area and Segmentation}

No relation between corpus callosum volume and oral reading was found, but corpus callosum area did have a statistically significant relation to reading in this sample. A regression of GORT-3 Passage scores on adjusted area at the midsagittal slice (with gender and IQ controlled) yielded statistically significant results, suggesting that taken together, the three independent variables accounted for about $27 \%$ of the within-family variance in reading achievement, $R^{2}=.267, F(3,40)=7.57, p<.001$. With gender and IQ controlled, the adjusted area of the corpus callosum at the midsagittal slice appeared to contribute moderately to reading scores. The change in $R^{2}$ was significant, with adjusted corpus callosum area added to the model, $\Delta R^{2}=.052, F(2,39)=4.574$, $p<.05$. A one-voxel increase in corpus callosum area is associated with a .043 increase in GORT-3 Passage standard scores $(b=$ $.043, \beta=.243$ ).

With area found to significantly contribute to within-family variance of GORT-3 Passage performance, considering the question of whether one particular portion of the area is relatively more important seemed a natural next step. Measurements for the five anterior-to-posterior segments of corpus callosum area adjusted for whole-brain volume were analyzed. See Figure 1 for a diagram of segmentation locations.

A regression with all five adjusted segments was performed to examine the relative association of each corpus callosum segment, with gender and IQ controlled. Multicollinearity was not excessive (variance inflation factor $<5$ ). Results suggested a moderate to large contribution of Segment $3(b=.665, \beta=.596)$. See Table 5 for segment coefficients. When Segment 3 was added to a model with all other segments, gender, and IQ, the change in $R^{2}$ was statistically significant, $\Delta R^{2}=.082, F(6,35)=7.683, p<.01$.

The GORT-3 Passage score is composed of scores for both reading rate and reading accuracy. To determine whether fluency or accuracy was more important in the relation between Segment 3 and oral reading, we performed separate regressions for GORT-3 Rate and Accuracy scores. Statistically significant results were obtained for both accuracy $(b=.571, \beta=.500, p<.05)$ and rate ( $b=.785, \beta=.704, p<.01)$, indicating that adjusted Segment 3 has a unique contribution to both aspects of reading apart from the rest of the corpus callosum. No other segments within the simultaneous regression contributed significantly to the within-family variance of accuracy or rate of reading.

Because the values for the Segment 3 contributions to GORT-3 Rate and Accuracy scores were obtained with two separate regressions, their relative importance is not directly comparable. To determine the relative importance of Segment 3 to rate and accuracy, we conducted a path analysis. The just-identified base model with standardized results is shown in Figure 2. The exogenous variables were allowed to correlate because they were correlated in the original regressions. The disturbances $(\mathrm{d} 1, \mathrm{~d} 2)$ for GORT-3 Rate and GORT-3 Accuracy scores were also correlated because it is likely that they share some variance not related to the model variables. To test for the relative importance of Segment 3 to Rate and Accuracy scores, the two paths from adjusted Segment 3 area to Rate and Accuracy scores were constrained to be equal in a second model. The change in chi-square relative to the change in degrees of freedom suggests no significant degradation of model fit, $\Delta \chi^{2}=1.285, \Delta d f=1, p=.257$. Segment 3 appears to have similar effects on GORT-3 Accuracy and GORT-3 Reading scores.

\section{Left-Right Volume Comparison}

The analyses of hemispheric differences in corpus callosum volume were studied as within individual differences, not withinfamily differences. Right- and left-side corpus callosum volumes were used as repeated measures in a one-way ANCOVA with fullscale IQ, gender, age, and diagnosis as covariates. The results of the test are shown in Table 6. They indicate that there are no significant differences in left and right corpus callosum volumes due to reading diagnosis, with full-scale IQ, gender, and age controlled.

\section{Midsagittal Slice and Corpus Callosum Volume}

A Pearson correlation adjusted for attenuation with the reliability estimates calculated for the corpus callosum volume and area measurements was used to test the correlation between the raw (unadjusted) voxel counts for area in the midsagittal slice and total corpus callosum volume. The correlation was found to be .878 , with a two-tailed significance of $p=.01$. Volume and area

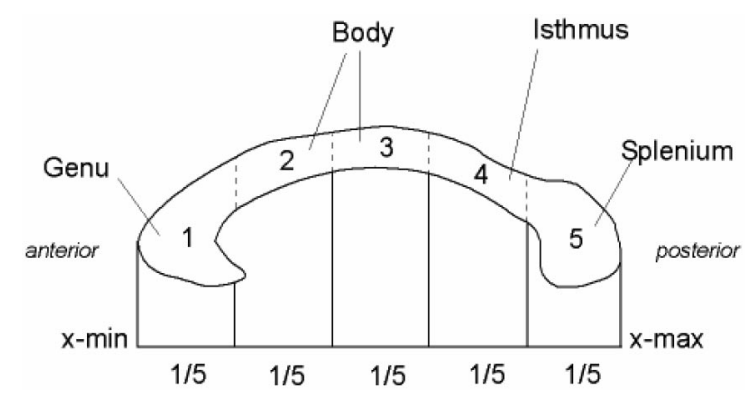

Figure 1. Segmentation of the midsagittal slice. $\mathrm{x}-\mathrm{min}=$ most anterior point; $\mathrm{x}$-max $=$ most posterior point. 
Table 5

Results of One Simultaneous Regression of GORT-3 Passage Scores on All Five Adjusted Segments of the Corpus Callosum, With Gender and $I Q$ controlled

\begin{tabular}{cccccc}
\hline Segment & $\begin{array}{c}\text { Unstandardized } \\
\text { coefficient } \\
(b)\end{array}$ & $\begin{array}{c}\text { Standard } \\
\text { error }\end{array}$ & $\begin{array}{c}\text { Standardized } \\
\text { coefficient } \\
(\beta)\end{array}$ & $\begin{array}{c}\text { Significance } \\
(p)\end{array}$ & $\begin{array}{c}\text { Effect size } \\
\left(\Delta R^{2}\right)\end{array}$ \\
\hline 1 & -.014 & .096 & -.025 & .881 & .001 \\
2 & .003 & .202 & .003 & .987 & .001 \\
3 & .665 & .240 & .596 & .009 & .082 \\
4 & -.205 & .150 & -.219 & .180 & .020 \\
5 & -.039 & .104 & -.074 & .711 & .001 \\
\hline
\end{tabular}

Note. GORT-3 = Gray Oral Reading Test-Third Edition.

measurements for this study are not entirely in accordance, but the correlation is high and statistically significant.

\section{Discussion}

\section{Why Area and Not Volume?}

Oral reading was not detectably variable in relation to corpus callosum volume within families in this study. However, corpus callosum area was found to contribute to the variance in oral reading such that better readers had larger corpus callosum areas. Both volume and area were measured with similar reliability. It may be that error accumulated in the volumetric measurements obscured a relatively small effect. Volumetric analysis includes the measurement of up to 20 sagittal slices, but area measurements are accomplished on a single midsagittal slice. Further, our measurements were anchored by the lateral limits of the ventricles, which vary widely in individuals. Although our measurements were reliable within individuals, error between persons was likely "reliably" measured, but constituted error nonetheless. Given the fairly high correlation between area and volume, the study results suggest that area may be a better unit of measurement for future studies.

\section{Segment 3 and Oral Reading}

Of the five segments of the corpus callosum area, it was the midbody segment that was most related to reading in the sample; it was larger in better readers. This finding was initially paradox- ical, as the perisylvian regions found to be involved in reading from a functional (fMRI) perspective (Shaywitz, 1998) are served by the posterior region of the corpus callosum, and it was here that results were expected. The midbody of the corpus callosum serves the motor functions of the brain including the oral-motor functions needed for word formation and the integration of primary and secondary sensory functioning (Aboitiz, Scheibel, Fisher, \& Zaidal, 1992),

One possible explanation of midbody result is that we are observing a downstream effect of poor early auditory temporal functioning. Lower level auditory sensory systems relying on temporal processing rates have been implicated in dyslexia such that longer times are required to discriminate sounds. Leppänen et al. (2002) found that by 6 months of age, infants with a high familial risk for dyslexia appear to process the duration of sound differently than infants not at risk. The authors conclude that, "those who go on to become dyslexic may have a deficit related to timing and perception of temporal cues in speech" (p. 419), which in turn might underlie deficits in phonological processing. Segment 3 , at the midbody of the corpus callosum, is presumed to be involved in the integration of primary sensory with higher level processing of auditory stimuli (Aboitiz et al., 1992), making it a likely place for differences to occur in people with severe RD.

Alternatively, or in addition, a larger midbody of the corpus callosum may reflect the specific nature of the measurement task: oral reading. Participants were asked to read aloud, requiring recruitment of both word formation and the physical reproduction of those sounds. The oral reading measurement task was composed

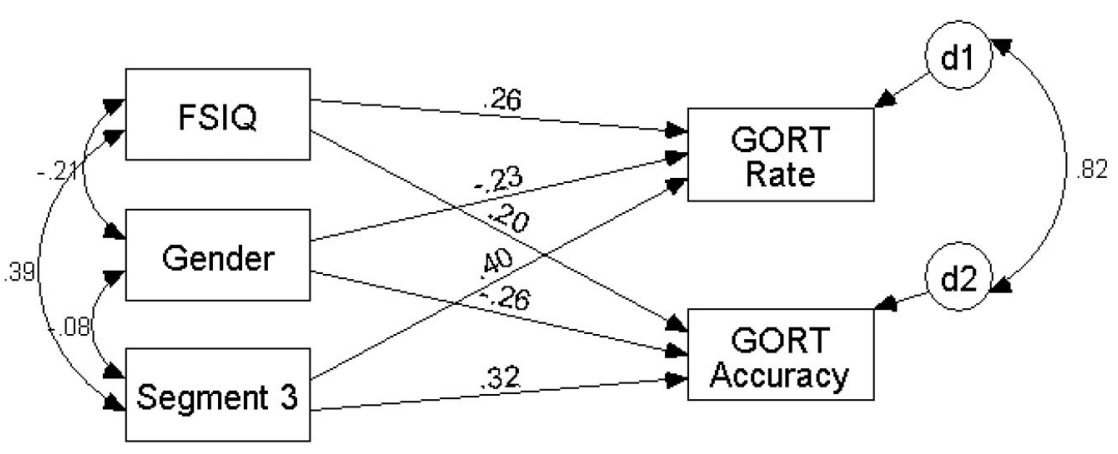

Figure 2. Path analysis model showing standardized results for the effect of adjusted Segment 3 on Rate and Accuracy scores on the Gray Oral Reading Test-Third Edition (GORT). FSIQ = full-scale IQ. 
Table 6

Results of a Mixed Repeated Measures Analysis of Covariance of the Difference in Right and Left Corpus Callosum Volume, With IQ, Gender, Age, and Diagnosis as Covariates

\begin{tabular}{|c|c|c|c|c|c|}
\hline Source & Sum of squares & Mean square & $F(d f)$ & $p$ & Partial eta squared \\
\hline \multicolumn{6}{|l|}{ Within subjects } \\
\hline Hemisphere (left-right) & $65,619.977$ & $65,619.977$ & $.077(1,63)$ & .783 & .001 \\
\hline Hemisphere $\times$ Diagnosis & $1,201,286.852$ & $1,201,286.852$ & $1.404(1,63)$ & .240 & .022 \\
\hline Error (hemisphere) & $53,900,886.257$ & $855,569.623$ & & & \\
\hline \multicolumn{6}{|l|}{ Between subjects } \\
\hline $\mathrm{IQ}^{\mathrm{a}}$ & $1,329,738.942$ & $1,329,738.942$ & $.373(1,63)$ & .373 & .001 \\
\hline Gender $^{\mathrm{a}}$ & $11,692,327.858$ & $11,692,327.858$ & $7.075(1,63)$ & .010 & .106 \\
\hline $\operatorname{Age}^{\mathrm{a}}$ & $499,199.711$ & $499,199.711$ & $.302(1,63)$ & .585 & .071 \\
\hline Diagnosis & $127,453.490$ & $127,453.490$ & $.077(1,63)$ & .782 & .022 \\
\hline Error (between) & $102,033,675.000$ & $1,729,384.323$ & & & \\
\hline
\end{tabular}

of both rate and accuracy measurements. However, Segment 3, at the midbody of the corpus callosum, appears to be similarly involved in both the rate and accuracy of reading in this study.

There is a body of research suggesting general temporal and motoric deficits in people with RD. A number of studies have found deficits in participants with dyslexia relative to normal readers in temporal order on matching for both visual, auditory, and cross-modal tasks (Lassonen, Tomma-Halme, Lahti-Nuuttila, Service, \& Virsu, 2000; Rose, Feldman, Jankowski, \& Futerweit, 1999). Poorer adult readers have been found to perform more slowly on visual temporal ordering tasks (Hari, Renvall, \& Tanskanen, 2001; May, Williams, \& Dunlap, 1988). Similarly, auditory deficits in temporal processing of speech perception in children have been found as well as motor deficits (Beaton, 2004). Although sensory-motor and auditory deficits have been recognized as often co-occurring in people with $\mathrm{RD}$, there is some disagreement about the importance of these deficits as a central feature of dysfunctional reading (Ramus et al., 2003). It is possible that the findings of this study represent characteristics common to those with sensory-motor and auditory problems in addition to a core phonological deficit.

\section{Limitations}

This study examined within-family differences, an approach that seems appropriate for a disorder known to be genetically inherited. The sample was limited by the very small number of adults with $\mathrm{RD}$ and by insufficient data on the amount of remediation received by participants with RD. Although parents share genes with their children, they do not share genes with each other, nor would they necessarily have shared similar family environments as children. A study involving only siblings might provide a more pure sample for controlling genetic and environmental differences; this study sample was too small to allow for this, and many families in the study included only one child. Still, it is important to note that bivariate correlations yielded stronger results once the data were centered about the family mean, indicating that using withinfamily variance may allow us to see relationships that are normally masked by variability among unrelated participants.

A major limitation of this study is that without concurrent functional data, we cannot associate brain structure with brain activity. The finding of a larger midbody of the corpus callosum in better oral readers within families could represent an effect having to do with something not directly related to reading or marginally related, such as the physical vocalization of sounds. Alternatively, the finding could represent an overarching effect such as faster cognitive processing in general. By closely coupling structural and brain activation data, say, during a reading activity, researchers can obtain better information regarding the neural substrates of reading.

\section{Conclusions}

The corpus callosum was found to have a moderate effect on reading in this study sample. Some variance, whether direct or the result of some broader function, exists such that better readers have larger areas of the corpus callosum at the midsagittal slice and specifically at the midbody. Left and right corpus callosum volumes are symmetrical and do not vary with RD diagnosis. Whether volume is required to study the corpus callosum is questionable. Our measurements were reliable for volume for remeasure of individuals, but individual variation in ventricle size may have introduced too much error.

Many of the childhood syndromes we study seem to have some degree of heritability, yet we rarely study them within families. This study demonstrates the utility of comparing family members for differences along a dimension of interest. The corpus callosum has very high variability among individuals, but when compared among family members in combination with behavioral data, results emerged. Studying families seems to be a rich approach affording natural controls for differences in environment that occur when comparing groups of unrelated individuals. We recommend this method for future research.

\section{References}

Aboitiz, F., Scheibel, A. B., Fisher, R. S., \& Zaidal, E. (1992). Fiber composition of the human corpus callosum. Brain Research, 598, 143153.

Beaton, A. A. (1997). The relation of planum temporale asymmetry and morphology of the corpus callosum to handedness, gender, and dyslexia: A review of the evidence. Brain and Language, 60, 255-322.

Beaton, A. A. (2004). Dyslexia, reading and the brain: A sourcebook of psychological and biological research. New York: Psychology Press. 
Breier, J. I., Simos, P. G., Fletcher, J. M., Castillo, E. M., Zhang, W., \& Papanicolaou, A. C. (2003). Abnormal activation of temporoparietal language areas during phonetic analysis in children with dyslexia. $\mathrm{Neu}$ ropsychology, 17, 1-12.

Castro-Caldas, A., Miranda, P. C., Carmo, I., Reis, A., Leote, F., Ribeiro, C., et al. (1999). Influence of learning to read and write on the morphology of the corpus callosum. European Journal of Neurology, 6, $23-28$.

Cox, R. W. (1996). MCW AFNI user manual. Milwaukee, WI: Medical College of Wisconsin.

Duara, B., Kushch, A., Gross-Glenn, K., Barker, W. W., Jallad, B., Pascal, S., et al. (1991). Neuroanatomic differences between dyslexic and normal readers on magnetic resonance imaging scans. Archives of Neurology, 48, 410-416.

Hari, R., Renvall, H., \& Tanskanen, T. (2001). Left minineglect in dyslexic adults. Brain, 124, 1373-1380.

Hynd, G. W., Hall, J., Novey, E. S., Eliopulos, D., Black, K., Gonzales, J. J., et al. (1995). Dyslexia and corpus callosum morphology. Archives of Neurology, 52, 32-38.

Hynd, G. W., Semrud-Clikeman, M., Lorys, A. R., Novey, E. S., \& Eliopulos, D. (1990). Brain morphology in developmental dyslexia and attention deficit disorder/hyperactivity. Archives of Neurology, 47, 919 926.

Hynd, G. W., Semrud-Clikeman, M., Lorys, A. R., Novey, E. S., Eliopulos, D., \& Lyytinen, H. (1991). Corpus callosum morphology in attention deficit-hyperactivity disorder: Morphometric analysis of MRI. Journal of Learning Disabilities, 24, 141-146.

Jenner, A. R., Rosen, G. D., \& Galaburda, A. M. (1999). Neuronal asymmetries in primary visual cortex of dyslexic and nondyslexic brains. Annals of Neurology, 46, 189-196.

Kinsbourne, M. (2003). The corpus callosum equilibrates the cerebral hemispheres. In E. Zaidel \& M. Iacoboni (Eds.), The parallel brain: The cognitive neuroscience of the corpus callosum (pp. 271-281). Cambridge, MA: MIT Press.

Kopcik, J. R., Juraska, J. M., \& Washburne, D. L. (1986). Sex and environmental effects on the ultrastructure of the rat corpus callosum. Society for Neuroscience Abstracts, 12, 1218.

Larsen, J. P., Hoeien, T., \& Oedegaard, H. (1992). Magnetic resonance imaging of the corpus callosum in developmental dyslexia. Cognitive Neuropsychology, 9, 123-134.

Lassonen, M., Tomma-Halme, J., Lahti-Nuuttila, P., Service, E., \& Virsu, V. (2000). Rate of information segregation in developmentally dyslexic children. Brain and Language, 75, 66-81.

Leppänen, P. H. T., Richardson, U., Pihko, E., Eklund, K. M., Guttorm, T. K., Aro, M., et al. (2002). Brain responses to changes in speech sound durations differ between infants with and without familial risk for dyslexia. Developmental Neuropsychology, 22, 407-422.

May, J. G., Williams, M. C., \& Dunlap, W. P. (1988). Temporal order judgements in good and poor readers. Neuropsychologia, 20, 917-924.
Njiokiktjien, C., de Sonneville, L., \& Vaal, J. (1994). Callosal size in children with learning disabilities. Behavioral Brain Research, 64, 213218.

Psychological Corporation. (1999). Wechsler Abbreviated Scale of Intelligence. San Antonio, TX: Harcourt Assessment.

Ramus, F., Rosen, S., Dakin, S. C., Day, B. L., Castellote, J. M., White, S., et al. (2003). Theories of developmental dyslexia: Insights from a multiple case study of dyslexic adults. Brain, 126, 841-865.

Robichon, F., \& Habib, M. (1998). Abnormal callosal morphology in male adult dyslexics: Relationships to handedness and phonological abilities. Brain and Language, 62, 127-146.

Rose, S. A., Feldman, J. F., Jankowski, J. J., \& Futerweit, L. R. (1999). Visual and auditory temporal processing, cross-modal transfer, and reading. Journal of Learning Disabilities, 32, 256-266.

Rumsey, J. M., Casanova, M., Mannheim, G. B., Patronas, N., DeVaughn, N., Hamburger, S. D., et al. (1996). Corpus callosum morphology, as measured with MRI, in dyslexic men. Biological Psychiatry, 39, 769775.

Rumsey, J. M., Dorwart, R., Vermess, M., Denckla, M. B., Druesi, M. J., \& Rappaport, J. L. (1986). Magnetic resonance imaging of brain anatomy in severe developmental dyslexia. Archives of Neurology, 43, 1045-1046.

Scamvougeras, A., Kigar, D. L., Jones, D., Weinberger, D. R., \& Witelson, S. F. (2003). Size of the human corpus callosum is genetically determined: An MRI study in mono- and dizygotic twins. Neuroscience Letters, 338, 91-94.

Schlaug, G., Jäncke, L., Huang, Y., Staiger, J. F., \& Steinmetz, H. (1995). In vivo evidence of structural brain asymmetry in musicians. Science, 267, 699-701.

Shaywitz, S. E. (1998). Dyslexia. The New England Journal of Medicine, 338, 307-312.

Shaywitz, S. E., Shaywitz, B. A., Pugh, K. R., Fulbright, R. K., Constable, R. T., \& Mencl, W. E. (1998). Functional disruption in the organization of the brain for reading in dyslexia. Proceedings of the National Academy of Science USA, 95, 2636-2641.

Snowling, M. J. (2000). Dyslexia (2nd ed.). Malden, MA: Blackwell.

Stevens, J. (2002). Applied multivariate statistics for the social sciences (4th ed.). Mahwah, NJ: Erlbaum.

Strauss, E., \& Wada, J. (1993). Callosal morphology and performance on intelligence tests. Journal of Clinical and Experimental Neuropsychology, 16, 79-83.

Wiederholt, J. L., \& Bryant, B. R. (1992). Gray Oral Reading Test (3rd ed.). Austin, TX: Pro-Ed.

Received December 14, 2005

Revision received June 14, 2006

Accepted September 1, 2006 